\title{
Cars as Roadside Units: A Self-Organizing Network Solution
}

\author{
Ozan K. Tonguz ${ }^{\dagger}$ and Wantanee Viriyasitavat ${ }^{\dagger \ddagger}$ \\ ${ }^{\dagger}$ Carnegie Mellon University, Dept. of Electrical and Computer Engineering, Pittsburgh, PA 15213-3890, USA \\ ${ }^{\ddagger}$ Faculty of Information and Communication Technology, Mahidol University, Thailand \\ E-mail: tonguz@ece.cmu.edu,wviriyas@ece.cmu.edu
}

\begin{abstract}
Deploying Roadside Units (RSUs) for increasing the connectivity of vehicular ad hoc networks is deemed necessary for coping with the partial penetration of Dedicated Short Range Communications (DSRC) radios into the market at the initial stages of DSRC deployment. Several factors including cost, complexity, existing systems, and lack of cooperation between government and private sectors have impeded the deployment of RSUs. In this paper, we propose to solve this formidable problem by using a biologically inspired self-organizing network approach whereby certain vehicles serve as RSUs. The proposed solution is based on designing local rules and the corresponding algorithms that implement such local rules. Results show that the proposed approach can increase the message reachability and connectivity substantially.
\end{abstract}

\section{INTRODUCTION}

Successful deployment of vehicular ad hoc networks (VANETs) where information (such as traffic, road information or safety messages) is sent, forwarded, and received by vehicles depends on the adoption of the new wireless technology, namely the Dedicated Short Range Communications (DSRC) technology. Since it is anticipated that the DSRC technology might be a mandate for modern vehicles effective 2017, with high probability, vehicle-toinfrastructure (V2I) communications-based networks will be the first type of vehicular ad hoc networks (VANETs) ${ }^{1}$ that might be implemented and, as such, they could accelerate the adoption of the DSRC technology.

Besides V2I applications (e.g., Internet Access), additional infrastructure can also be used to improve connectivity of vehicle-to-vehicle (V2V) networks. In addition to growing demand for $\mathrm{V} 2 \mathrm{X}$ traffic $^{2}$ and the fact that V2V applications are confined to a particular geographical area, installing special Roadside Units (RSUs) has emerged as an attractive solution (especially to the Department of Transportation) for providing infrastructure support as RSUs limit information to be disseminated within a confined area, thus resulting in smaller message delay, better information security, and possibly lower communications cost.

While RSUs seem to be a very promising solution for improving V2V communications, the cost of manufacturing, installing, and maintaining these units seem to be prohibitive for the large-scale deployment of RSUs. For example, a simplistic form of RSU (such as Roadway Probe Beacons) requires $\$ 13,000-15,000$ per unit capital cost and up to $\$ 2,400$ per unit per year ${ }^{3}$ for operation and maintenance [1]. In addition to cost, effectiveness and utilization rate of RSUs may also depend on the number of DSRC-equipped vehicles that are present in a given area. As an example, consider an accident notification message; RSUs will be utilized only for vehicles within a small region that is relevant to the notification message, typically a 4 square miles area around the accident scene.

\footnotetext{
${ }^{1}$ VANETs could be based on vehicle-to-infrastructure (V2I) and/or vehicle-to-vehicle (V2V) communications.

${ }^{2} \mathrm{~V} 2 \mathrm{X}$ is an abbreviation used for both V2I and V2V communications.

${ }^{3}$ The price quote includes the cost of both roadside equipment and roadside wireless communications.
} 
In this paper, based on a new biologically-inspired networking paradigm, we propose to leverage the initially available DSRC-equipped vehicles to be used as temporary RSUs. Vehicles that act as temporary RSUs can make brief stops during which they act as a communication bridge for other vehicles in the network [2], [3]. We envision that using vehicles as RSUs could improve not only the message reachability and network connectivity but also accelerate the adoption of DSRC technology in addition to avoiding the cost of deploying Roadside Units.

\section{Problem Statement}

The U.S. Department of Transportation (DoT) was expected to have a nationwide deployment of the roadside infrastructure in 2008 [4]. This plan, however, did not materialize and, to date, very few RSUs have been deployed. Major reasons that prevented the success of the plan can be summarized as follows:

\section{A. Justifying the benefits that RSUs provide is difficult}

Determining the value of such a radical proposition in uncertain future markets has proven to be nontrivial and fairly complicated. Even though the benefits of V2V and V2I systems in terms of safety, traffic efficiency, and environment are clear and have been reported in [5], the full benefits of the DSRC technology can not be realized unless the technology is widely adopted by the market. Such economic justification becomes more difficult when there are other traffic information infrastructures such as Dynamic Message Sign (DMS) and 5-1-1 System which are already in place [6]. These existing systems provide both safety and traffic efficiency benefits (i.e., roadside assistance help alert drivers of slow vehicles ahead and/or upcoming work zone while a transportation and traffic information telephone hotline allows travelers to choose the most efficient mode and route to their final destination). Proven effectiveness, high user satisfaction [6], existing widespread deployment, and already-invested capital ${ }^{4}$ into the existing systems have further impeded the nationwide deployment of RSUs.

\section{B. Global cooperation and partnership with private sector}

The deployment of roadside infrastructure (i.e., through the DoT's VII program) requires major collaboration and coalition of public and private sectors (such as US DOT, state and city authorities, auto companies, and other profit and non-profit organizations). However, the willingness of public- and private-sectors to cooperate in this effort is a major issue as privacy, ownership, funding, and use are all major concerns. Until now, this cooperation which is a prerequisite for the success of roadside infrastructure deployment, as reported in [7], has remained largely unconsummated.

\section{Funding approaches}

One of the most important stumbling blocks in the infrastructure deployment is funding. The DoT long-term deployment plan which envisioned 200,000 - 250,000 roadside units to be installed [4] potentially require billions of dollars of investment. Current uncertain economic climate as well as the previously mentioned difficulties in

\footnotetext{
${ }^{4}$ Deployment cost of 5-1-1 system ranges from $\$ 133,000$ to $\$ 1,028,000$ [6].
} 
justifying the benefits of this new technology and lack of a healthy cooperation between different organizations have crippled the initiative required for financing the deployment program.

It is interesting to note that most of the above reasons are non-technical in nature. Social, economic, and political issues are major obstacles that have impeded the extensive deployment of RSUs. Since major collaborative efforts are necessary to resolve these issues, in this paper, we propose an alternative approach to roadside infrastructure by leveraging the use of existing DSRC-equipped vehicles to provide RSU functionality. This approach employs a powerful self-organizing network paradigm and draws its inspiration from social insect colonies such as ants, bees, birds, and fish. Such an approach was formulated for the first time in [8] where it was suggested that several fundamental transportation problems can be solved by carefully studying the behavior of self-organizing biological systems and applying the underlying principles of their successful operation to transportation problems. In [8], as an example of this approach, it was shown that vehicular traffic at intersections in urban areas can be managed by a new technology known as "Virtual Traffic Lights" via the cooperation of vehicles at that intersection through vehicle-to-vehicle communications and without the need for infrastructure-based traffic control. In this paper, we show that another acute transportation problem (namely, the deployment of RSUs) can also be solved by using the same powerful approach.

\section{Proposed Solution: CARS AS RSUs}

Inspired by social insect colonies such as ants, bees, and termites, we propose a self-organizing network solution that leverages the existing DSRC-equipped vehicles to be used as temporary RSUs. As a temporary RSU, a vehicle can make a brief stop and take on or assume the tasks of a conventional RSU - relaying messages to nearby vehicles and acting as a communication bridge for other vehicles in the network. The proposed solution consists of 2 main components, each of which addresses the following key questions:

1) Which vehicles should act as temporary RSUs? How can a vehicle determine, in an independent and distributed manner, whether or not it should temporarily serve as an RSU?

2) What are the tasks of temporary RSUs? What does a vehicle have to do while serving as a temporary RSU? How long should it continue to serve as an RSU?

In order to answer the above questions, one needs to have a target application. Different applications may require different algorithms/solutions to the aforementioned questions. To demonstrate the feasibility of the proposed concept, a specific safety application, namely a Post Crash Notification (PCN) application, is used in this paper as an illustrative example to show how the proposed "poor man's solution", with zero infrastructure support, can help improve the network connectivity. The main purpose of the PCN application is to disseminate a safety message (i.e., information about the incident - time, location, etc - which may be issued by a vehicle involved in the accident or a police car) to all vehicles within a region of interest (ROI) and the message should be disseminated to these vehicles within a short amount of time. The following subsection describes in detail how the proposed solution can be implemented. 


\section{A. Distributed algorithm for selecting a temporary RSU}

Figure 1(a) presents a simple example that provides important insights into how the RSU-selection algorithm should be designed. A flow diagram of the proposed algorithm is shown in Figure 2.

Consider an example of a network as shown in Figure 1(a); black, green, and blue squares and red circle represent vehicles whereas arrows represent movement direction of vehicles. Assume, with no loss of generality, that an accident takes place at the center of this network and the Vehicle Src involved in the accident sends out a post crash notification message (i.e., a safety message) to other vehicles in the network. After the first broadcast from $S r c$, all vehicles in the gray-shaded region (i.e., the coverage polygon) receive the message and are informed about the accident. Note that the coverage polygon is the polygon that contains all vehicles that are informed via spatial relays from Src or other informed vehicles. In other words, the polygon contains all vehicles that could be reached from Src either via direct transmission or via multi-hop forwarding. Based on the definition of the coverage polygon, the following observations can be made:

Observation 1: Good candidates for temporary RSUs are vehicles that are positioned at the boundary of the coverage polygon.

By definition, vehicles that are on the boundary of the network polygon have both informed and uninformed vehicles in their vicinity ${ }^{5}$. These vehicles are therefore highly likely to meet with other uninformed vehicles before other non-boundary and informed vehicles meet uninformed ones. The nonboundary and informed vehicles, on the other hand, are mostly surrounded by informed vehicles; hence there is no additional benefit in having these vehicles serve as temporary RSUs. Figure 1(a) provides a simple example - coverage polygon is shaded in gray color, and blue and green rectangles represent boundary and non-boundary vehicles, respectively.

Observation 2: Only boundary vehicles that travel toward the accident should serve as temporary RSUs.

In addition to the position of vehicles, movement directions of these vehicles should also be considered as well. In this paper, based on the mobility pattern assumed, we propose that only boundary vehicles that travel toward the accident should stop and serve as temporary RSUs. By having these vehicles stop at their current locations for a brief period of time (and not continue to travel toward the accident scene), the subsequent rebroadcasts from these vehicles could possibly reach other uninformed vehicles when they arrive into the RSUs' neighborhood. It should be noted that the boundary vehicles that travel in the outward direction from the scene of accident do not stop; message can be disseminated quickly through spatial relays of these vehicles that store, carry, and later forward (SCF) the message to vehicles that are in a region these vehicles travel toward (i.e., this region that outside the coverage polygon. In Figure 1(a), only vehicle $C$ (not vehicles $B, E, F, G$ or $I$ ) will act as a temporary RSU. In the remainder of this paper, we will refer to rebroadcast from temporary RSUs (e.g., Vehicle $C$ ) as RSU rebroadcasts and

\footnotetext{
${ }^{5}$ Vicinity in this case implies nearby region and is not equivalent to the term neighborhood.
} 
rebroadcasts from other vehicles as SCF rebroadcasts.

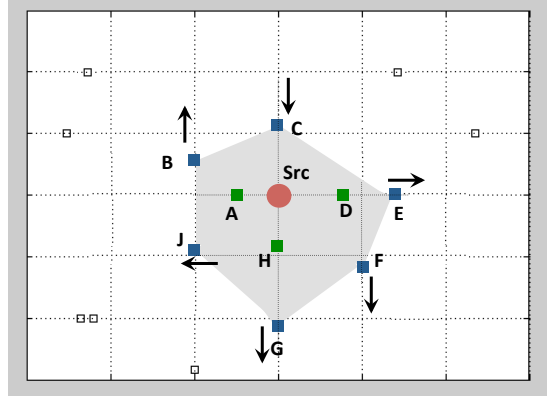

(a)
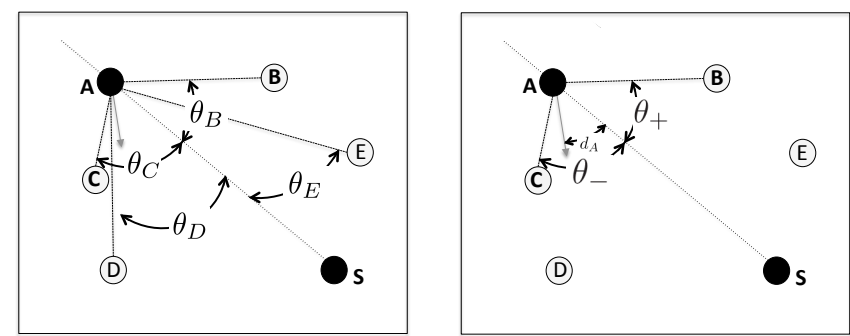

(b)

Fig. 1. (a) Boundary of the network coverage polygon. Assume that the message is broadcast from Src at the scene of accident. Vehicles that are connected to $S r c$ via spatial relays are in the gray shaded region. Boundary and non-boundary vehicles are indicated with blue and green dots, respectively. Black squares indicate vehicles that do not receive spatial message broadcast from $S r c$. Black arrows indicate current direction of the vehicles; (b) The principle of operation of the proposed distributed gift-wrapping algorithm. Assume that NBR $(A)=$ $\{S, B, C, D, E\}$, moving direction of $A$ is indicated by the gray arrow (in Figure 1(b)), and that $A$ first receives the message from Vehicle $S[9]$.

In order to determine an accurate coverage polygon and its boundary, one needs global knowledge of the network (i.e., location of all vehicles in the network). However, since such information requires excessive information exchange between vehicles which is not desirable in VANETs, we use the distributed gift-wrapping algorithm proposed in [9]. This algorithm is a distributed algorithm; a vehicle, upon receiving a message, can determine independently and in a distributed manner whether it lies on the boundary of the coverage polygon. Note that since it only relies on the local information, the distributed gift-wrapping algorithm is only an approximate algorithm and it tends to over-select boundary vehicles (i.e., some vehicles selected by the algorithm may not lie on the boundary of the polygon). More details about this algorithm can be found in [9]. In addition to the gift-wrapping algorithm, additional rules that consider directions of vehicles are added to the original distributed gift-wrapping algorithm. The resulting algorithm shown in Algorithm 1 is then used to select vehicles to serve as temporary RSUs.

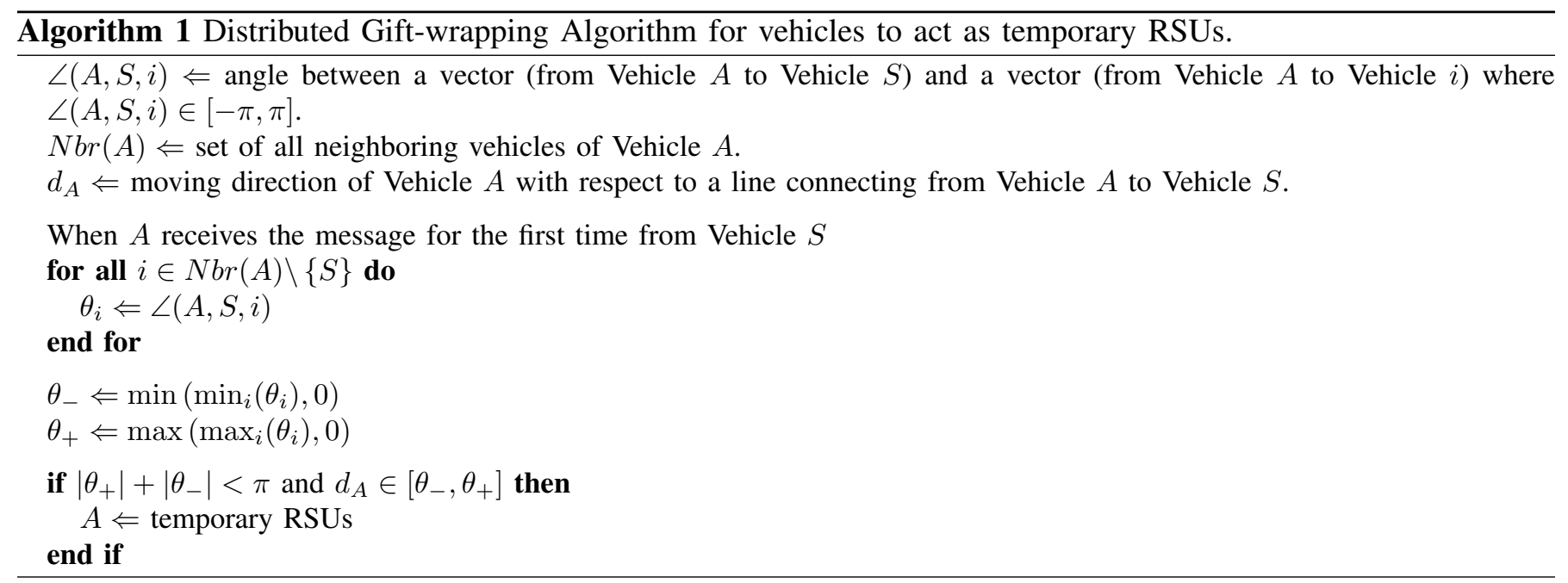

Figure 1(b) illustrates how the temporary RSUs are selected by Algorithm 1. Upon receiving a message for the 
first time from Vehicle $S$, Vehicle $A$ computes the angle $\theta_{i}$ for all of its neighbors (see Figure 1(b) (left)). Maximum $\left(\theta_{+}\right)$and minimum $\left(\theta_{-}\right)$angles are then identified. In the scenario given here, Vehicles $B$ and $C$ are the neighbors of Vehicle $A$ that have the maximum and minimum angles, respectively and since $\left|\theta_{+}\right|+\left|\theta_{-}\right|$is less than $\pi$ and moving direction of Vehicle $A$ falls between $\theta_{-}$and $\theta_{+}$, Vehicle $A$ is selected as a temporary RSU.

\section{B. Tasks of a temporary RSU}

As shown in the flow diagram in Figure 2, informed vehicles that are on the boundary of coverage polygon and moving toward the scene of accident act as temporary RSUs for a certain period of time. These vehicles make a brief stop and periodically rebroadcast the safety message to mimic the role of the conventional roadside units. Vehicles that receive such message rebroadcast from these temporary RSUs (i.e., RSU-vehicles) follow the same procedure as shown in Figure 2. It must be noted that when one considers a different (possibly non-safety) application such as instant messaging, content download, etc, the tasks of temporary RSUs may be changed - the temporary RSUs may stop for a different amount of time depending on the application; their stop duration may be preempted if the applications they support end; or instead of rebroadcasting the safety message, they may need to forward the messages to only particular vehicle(s).

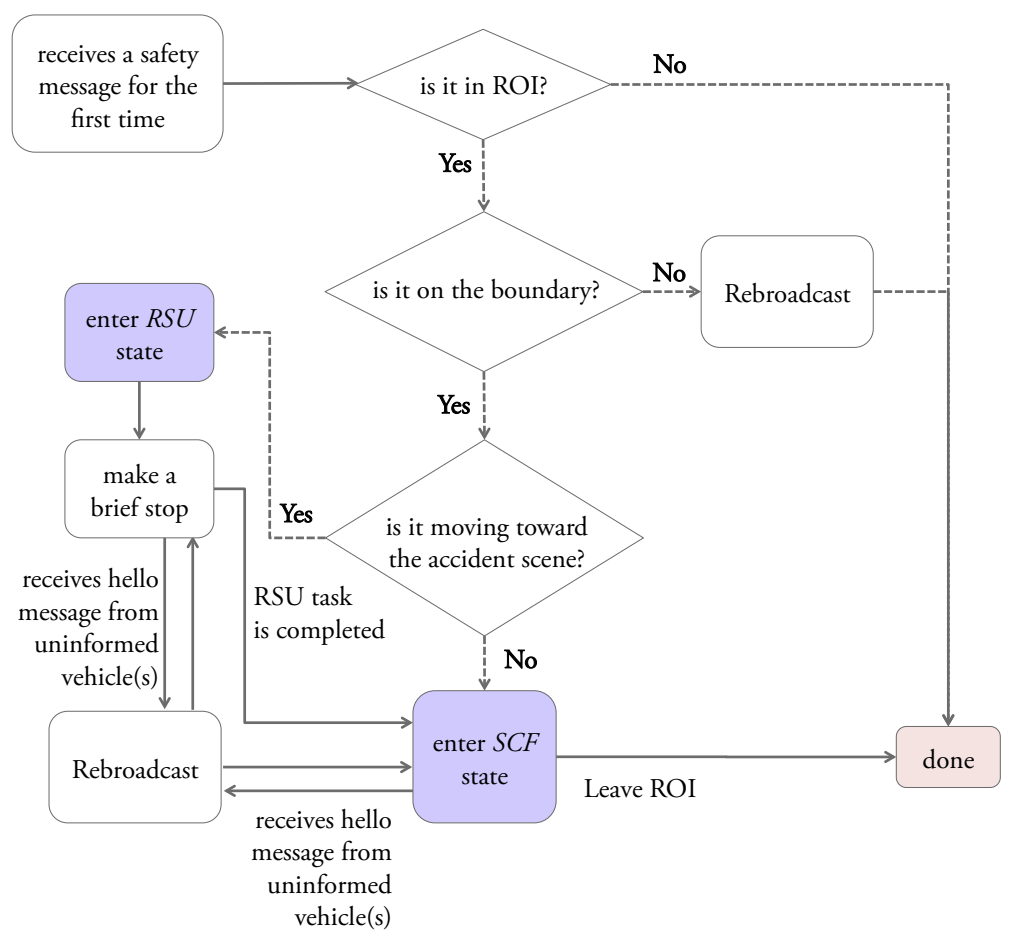

Fig. 2. Flow diagram describing the distributed algorithm for selecting a temporary RSUs and the tasks performed by temporary RSUs.

\section{Simulation Setting}

Traffic mobility model used in the simulations is based on the CA-based mobility model developed in [10] and parameter values used in the simulations are summarized in Table I. In the simulator, to maintain a constant vehicle 
density in the network, a new vehicle is immediately added to the network once a vehicle exits. We assume that this new vehicle is uninformed (i.e., it does not receive the safety message from prior rebroadcasts of the message).

TABLE I

PARAMETER VALUES FOR SimUlation STUdy.

\begin{tabular}{lc}
\hline \hline Parameters & Values \\
\hline Size of the road structure & $0.5 \mathrm{~km} \times 0.5 \mathrm{~km}, 1 \mathrm{~km} \times 1 \mathrm{~km}$ \\
Length of a road block & $2 \mathrm{~km} \times 2 \mathrm{~km}, 3 \mathrm{~km} \times 3 \mathrm{~km}$ \\
Vehicle density & 125 meters \\
DSRC penetration rate & $100 \mathrm{veh} / \mathrm{km}^{2}$ \\
Simulation time & $\{10 \%, 20 \%, \ldots, 100 \%\}$ \\
Maximum speed & 15 minutes $(5-$ minutes warmup period $)$ \\
Cycle duration & $15 \mathrm{~m} / \mathrm{s}(36 \mathrm{~km} / \mathrm{h})$ \\
Green light ratio & $45 \mathrm{~seconds}$ \\
Signal offset & $50 \mid 50$ \\
Stop time of RSU-vehicles & 10 seconds \\
\hline \hline
\end{tabular}

\section{A. Traffic Pattern}

Based on the commuting pattern in highly populated cities such as New York City (NYC), we observe that the traffic pattern can be categorized into four categories: (i) Morning Rush Hour Traffic (8-10 am); (ii) Lunch Hour Traffic; (iii) Evening Rush Hour Traffic; (iv) Midnight Traffic (1-3 am).

In the mobility model used in this paper, we assume the evening rush hour traffic where most of traffic travel in northbound direction. We also assume that the scene of accident is located at the top-center of the network and the safety message broadcasted by the source should be disseminated to all vehicles in the region.

\section{B. Transmission Range}

To take into account the possible obstruction of signal propagation due to buildings and highrises in urban cities, we assume two different types of communications: direct line-of-sight (LOS) and a non line-of-sight (NLOS) communications. LOS and NLOS have transmission ranges of 250 and 140 meters, respectively. Any two vehicles can communicate directly (i.e., in single-hop fashion) if and only if they are within the corresponding transmission ranges. In addition, we assume accurate GPS information in our simulations (i.e., a vehicle has perfect knowledge of positions of itself and all of its one-hop neighbors).

\section{Metrics}

In this paper, two performance metrics are used to evaluate the advantages of the proposed solution. Since the post crash notification application is considered as an example in this paper, message reachability metric is used to indicate the message dissemination rate achieved by the proposed solutions; i.e., fraction of vehicles that are informed when the proposed solution is implemented. It should be mentioned here that improvement in network connectivity comes at the expense of a slight increase in travel time. Vehicles that act as temporary RSUs need to make brief stops, resulting in an increase in travel time. To capture such an effect, average velocity of vehicles is considered. 
1) Message Reachability Metric: We use message reachability metric to evaluate the performance of our scheme against the standard scheme (i.e., no vehicles stop and serve as RSUs). Message reachability metric is defined as the fraction of vehicles in the network that receive the message. Note that the message reachability is different from network reachability metric. In other words, while the network reachability measures the maximum number of vehicles that are connected at a given point in time (i.e., a static metric) ${ }^{6}$, message reachability metric is a transitive measure of network reachability (see [11]).

2) Average vehicle velocity: Average vehicle velocity is used to capture how much the RSU's stop time affect the overall traffic flow in a city. Both average velocity of all vehicles and only those vehicles who act as temporary RSUs are reported.

\section{Simulation Results}

The simulation results are shown in Figure 3. Observe that the proposed "Cars as RSUs" scheme considerably outperforms the standard scheme; with the proposed scheme, the message reaches almost twice the number of vehicles in the network (i.e., the message reachability improves from $34 \%$ to almost $67 \%$ in a $100 \mathrm{veh} / \mathrm{km}^{2}$-dense network and $20 \%$ DSRC penetration rate, which corresponds to a $97 \%$ improvement).

Such an improvement is mainly due to the fact that with RSU scheme, vehicles that serve as RSUs stay in the network for a longer period of time (i.e., ratio of informed vehicles is higher) and since there are more informed vehicles (i.e., vehicles that have received the message), there are more message rebroadcasts which reach the uninformed vehicles (i.e., vehicles that have not received the message) with a higher probability. Note that the increase in network connectivity of the proposed scheme comes at the expense of a slight degradation in travel time (i.e., decrease in average vehicle velocity). Since vehicles make brief stops occasionally, our simulations have shown that velocity of a vehicle, on average, decreases by $0.27 \mathrm{~km} / \mathrm{h}$. This translates to a $1.48 \%$ decrease in average vehicle velocity and $1.51 \%$ increase in travel time. This, however, is a small increase as compared to the increase in travel time due to accident-induced congestion. It is worth noting that although the proposed scheme might not significantly outperform the standard scheme especially in the scenario with only $10 \%$ penetration rate, it is able to keep the safety message in the ROI for a long period of time (i.e., more than 300s). Observe that with the standard scheme, the informed vehicles spend little time in the ROI and are likely to leave the ROI before they are able to rebroadcast the message to other uninformed vehicles. As a result, the message reachability decreases with time and the message dies out after some time (i.e., 200s after the first broadcast in our case).

Figure 3(left) shows the reachability of networks with and without RSU-vehicles for different DSRC penetration rates. Observe that significant improvement in terms of network reachability can be achieved when RSU-vehicles are implemented only in a network with sparse and moderately-dense DSRC-equipped vehicles (i.e., 10\% - 40\% penetration rate). The improvement is most pronounced in moderately-dense network; i.e., a network with a certain density. When a network has very few DSRC-equipped vehicles, not much improvement is reported since RSU's

\footnotetext{
${ }^{6}$ which is equal to the fraction of vehicles that belong to the largest connected component of a network
} 

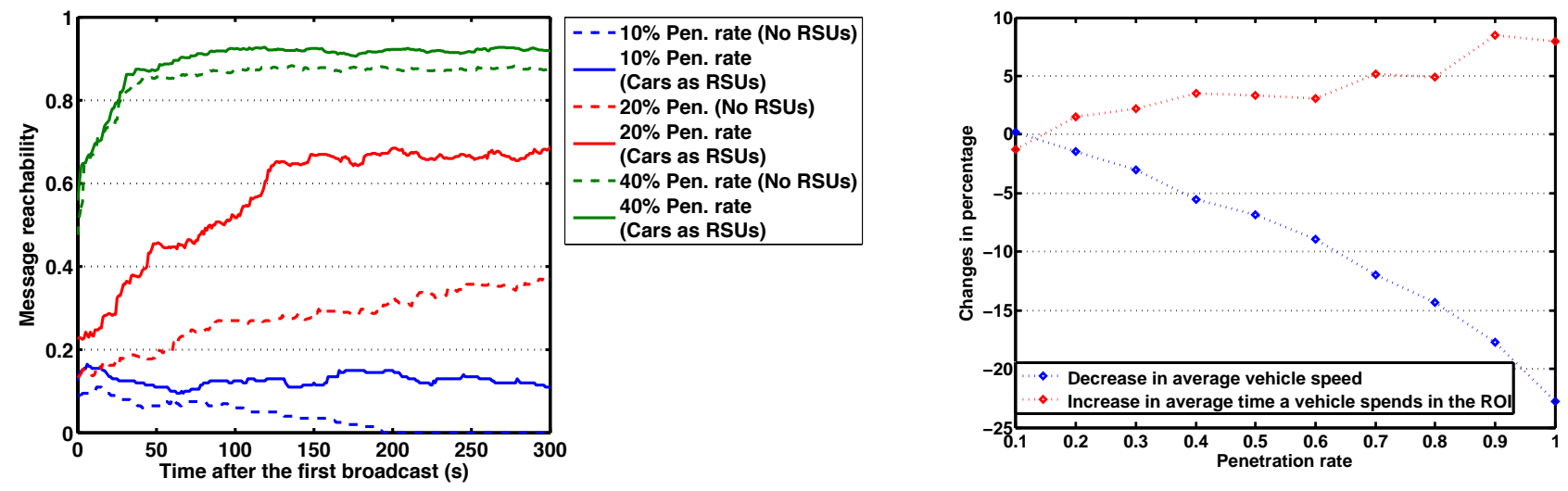

Fig. 3. Impact of vehicle density on the message reachability (left figure) and average vehicle speed and average time a vehicle spends in the ROI (right figure) when the proposed scheme is implemented. Substantial improvement is observed only in sparse and moderately-dense DSRC-equipped network. The results are based on a $1 \mathrm{~km}$ x $1 \mathrm{~km}$ Region of Interest and 30s of RSU-vehicle stop time.

stop time may not be long enough for the temporary RSUs to encounter other uninformed vehicles. In addition, it should be noted that, in a very sparse network, the coverage polygon (see Figure 1(a)) usually has a small size. Vehicles that act as temporary RSUs may be located very close to the accident. Although an uninformed vehicle receives a safety message from one of these RSUs, the message may no longer be useful as they are already close to the accident scene, having passed the last exit or alternative route that exists before driving into the congestion induced by the accident. On the other hand, when there are many DSRC-equipped vehicles in the network (i.e., a DSRC-equipped-dense network), the network is already well-connected and no vehicle is needed to act as temporary RSUs. By having some vehicles in a DSRC-equipped-dense network stop as RSUs not only degrades the message reachability but also impedes the overall traffic flow. Figure 3(right) shows almost $8 \%$ increase in average time a vehicle spends in the ROI and $24 \%$ decrease in average vehicle speed when the network is dense.

While message reachability may increase with the vehicle density (since dense traffic leads to a decrease in vehicle speed), our simulation results show that a change in the total number of vehicles in the network (i.e., density of equipped and unequipped vehicles combined) has far less effect on message reachability as compared to the impact caused by a change in the number of DSRC-equipped vehicles.

\section{EFFECT OF DifFERENT PARAMETERS}

\section{A. Effect of the size of Region of Interest (ROI)}

Our simulation results show that the message reachability of both schemes decreases with the size of the Region of Interest (ROI). This is due to the fact that size of the coverage polygon (see Figure 1(a)) of networks with the same density does not change with the size of ROI. Since message reachability metric is a relative measure, it follows that the fraction of vehicles that are in the coverage polygon (hence, the message reachability) decreases when the size of ROI increases. In addition, when one considers additional rebroadcasts from the temporary RSUs, 
similar conclusions can be drawn: the number of uninformed vehicles that are informed by the rebroadcasts from these RSUs does not vary with the ROI size; hence, the message reachability is higher when the ROI size is small. For example, for a density of $100 \mathrm{veh} / \mathrm{km}^{2}, 20 \%$ DSRC penetration rate, and $30 \mathrm{~s}$ RSU-vehicle stopping time, message reachability 2 minutes after the broadcast increases from $27 \%$ to $60.75 \%$ for $1 \mathrm{~km} \times 1 \mathrm{~km}$ ROI, whereas for a $3 \mathrm{~km}$ x $3 \mathrm{~km}$ ROI, it increases from $6.39 \%$ to $15.35 \%$.

\section{B. Effect of Stop time of RSU-vehicles}

Figure 4 shows the significant effect of stop time of RSU-vehicles on the message reachability. Observe that when the stop time is too short, RSU-agent scheme gives comparable performance to the conventional scheme without the RSU-vehicles. This is because the RSU-vehicles do not stop long enough to encounter other uninformed vehicles. As a result, the message reachability increases with the stop time until the stop time reaches a certain value (i.e., 30 seconds in this case). Any additional stop time beyond 30 seconds does not improve the message reachability because the RSU-vehicles are unlikely to encounter uninformed vehicles after having stopped for some time (i.e., 30 seconds in this case). It is therefore more beneficial for these vehicles to continue to move and possibly further rebroadcast the message to uninformed vehicles in other areas in the region of interest. Note also that too large stop time not only degrades the message reachability, but it also increases the travel delays of the vehicles that act as temporary RSUs. Note that the optimal stop time depends both on the vehicle density, size of Region of Interest (ROI), and topology of the network. While 30 seconds seems to be the optimal stop time for the network with $20 \%$ DSRC penetration rate (see Figure 4(a)), a smaller stop time (i.e., 20 seconds of stop time) would be needed for the maximum message reachability at $40 \%$ DSRC penetration rate network (see Figure 4(b)).

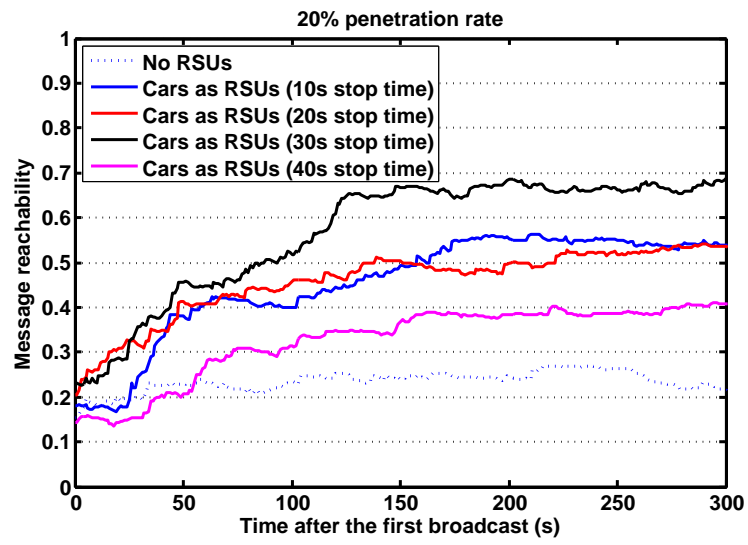

(a)

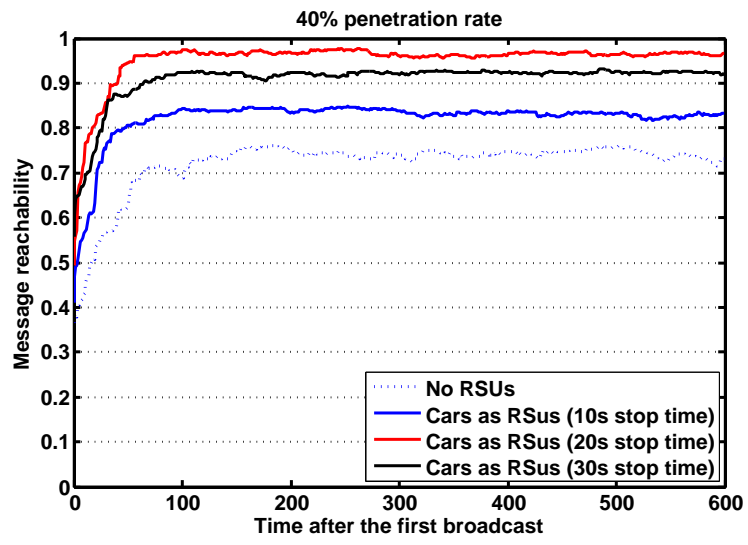

(b)

Fig. 4. Impact of the RSU-vehicle stop time on the message reachability of the network with and without RSU-vehicles. Substantial improvement is observed only in sparse and moderately-dense network. The results are based on a $1 \mathrm{~km} \times 1 \mathrm{~km}$ Region of Interest and vehicle densities of $100 \mathrm{veh} / \mathrm{km}^{2}$ with $20 \%$ and $40 \%$ DSRC penetration rates on the left and right figures, respectively. 


\section{Effect of Mobility Pattern}

Figure 5 shows the significant effect of mobility pattern of the vehicles on the message reachability. The proposed RSU-vehicle scheme provides significant improvement when most of the traffic move toward one particular direction; as the fraction of northbound traffic increases, the improvement of the RSU scheme also increases. For example, when $85 \%$ of traffic is northbound, the message reachability increases from $34 \%$ to $67 \%$ which corresponds to an improvement of $97 \%$. This suggests that the proposed RSU-scheme will work very well during the rush hours. For example, during the evening rush hours in Manhattan, a large fraction of people commute from their workplaces in downtown to their homes in the uptown area (i.e., dominant northbound traffic).

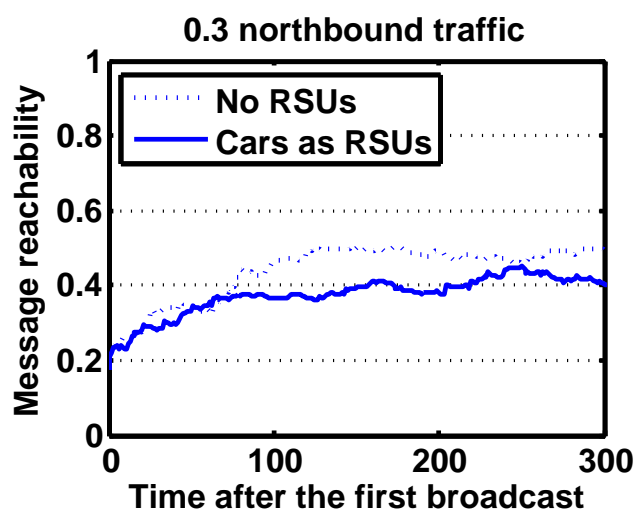

(a)

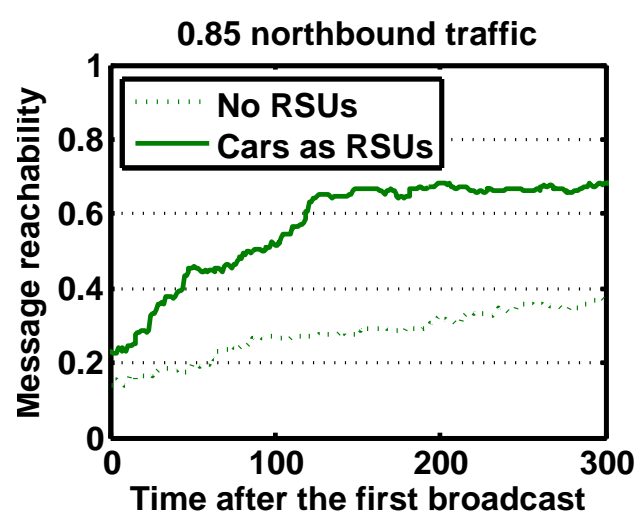

(b)

Fig. 5. Impact of the mobility pattern on the message reachability of the network with and without RSU-vehicles. The improvement of RSU-vehicle scheme over the conventional scheme increases with the increase in northbound traffic. The results are based on a $1 \mathrm{~km} x 1$ $\mathrm{km}$ Region of Interest network with vehicle density of $100 \mathrm{veh} / \mathrm{km}^{2}, 20 \%$ DSRC penetration rate, and 30-second RSU-vehicle stop time.

\section{DISCUSSION}

Consider the current way emergency situations are handled in urban areas: e.g., if there is an ambulance or fire truck trying to reach an emergency area, all the vehicles on the road move to the side of the road making way for the ambulance or firetruck to pass. This clearly delays all the vehicles agreeing to do this sacrifice as they slow down and move to the side of the road which probably takes anywhere from 30-60 seconds. This collective sacrifice can save the life of a sick patient or people who are trapped in a building on fire. In this paper, we use this example as a motivation for the proposed solution: a vehicle sending a safety message waits for an implicit acknowledgment before continuing its trip. If the ACK is not received, then the vehicle stops and keeps broadcasting the message till it receives an ACK (or until the scheduled broadcast duration ends). This way, the vehicle serves as a temporary RSU, thus enhancing the message reachability and network connectivity substantially.

The proposed system is extremely attractive due to its cost-effectiveness and its ubiquity, especially in the current environment where the deployment of roadside infrastructure is very limited. In a sense, the proposed solution is “a poor man's solution" to a very real and acute problem, namely the projected low penetration rates of the DSRC technology at the initial stages of DSRC deployment. As such, the main idea behind the proposed solution is to exploit the self-organizing network characteristics of a vehicular network whereby the fleet of cars can solve a 
formidable problem by themselves through cooperation without additional equipment or infrastructure. By using certain vehicles as temporary roadside units, this temporary infrastructure could be provided ubiquitously without requiring additional equipment. This approach not only improves connectivity of vehicular networks in the early stages of DSRC development, but it can also accelerate the adoption of the DSRC technology. For the successful operation of the proposed solution the following issues will need to be addressed: i) Cooperation from state or local traffic management institutions (an example of this is Zipcar [12]); ii) guaranteeing the correct and reliable operation against faults and/or malicious attacks; iii) incentives or other mechanisms for policy enforcement; iv)being able to use other alternatives to ZipCar (such as as taxis, buses, etc.); and v) penetration rate of DSRC technology. Our ongoing work is focused on addressing these issues.

While the cooperative approach presented here might appear altruistic for current day driving practices, it is not difficult to see that the presented approach could also be used by autonomous vehicles of the future. It is well-known that companies like Google and several car manufacturers such as GM, Volkswagen, Nissan etc. are currently looking into autonomous vehicles as a potential new technology. The authors of this paper believe that in the long run autonomous vehicles will have to use radios (such as DSRC radios or a similar communications technology) for $\mathrm{V} 2 \mathrm{~V}$ communications as this has tremendous benefits for even autonomous vehicles. The approach presented in this paper could thus be used for autonomous vehicles as well in which case human decisions will not be required and the car selected to serve as an RSU will do this automatically.

\section{RELATED WORK}

A comprehensive comparison between different type of RSUs is presented in [13]. Both analytical and simulation results reveal that relay and mesh nodes, as opposed to base stations, can be more cost-effective solutions even though a much larger number of such units are required to deliver the same level of performance as offered by the base stations. In addition, the authors also suggest that adding a small amount of infrastructure is vastly superior to even a large number of mobile nodes capable of routing to one another.

An interesting paper by Trullols et al. [14] uses the approach employed by the maximum coverage problem for addressing this issue. The authors identify that roadside infrastructure should be placed at intersections rather than the middle of road segment. Two strategies on the RSU placement locations are proposed: one that maximizes the number of vehicles served by RSUs, and the other that maximizes the number and the contact time between vehicles and RSUs. While the above studies are based on simulation data, a realistic trace of traffic is used to evaluate different schemes for RSU placement in [15]. The authors use a greedy algorithm to determine the minimum number and locations of RSUs that can serve all vehicles in Jeju city, Korea.

Several studies address the issue of effective communications between vehicles and RSUs. For example, a RSUbased solution for Collision Warning System (CWS) in urban areas is suggested in [16]. The authors propose an algorithm to determine when the RSUs installed at intersections should broadcast warnings to vehicles proceeding to the intersection. Zhang et al. propose in [17] a scheduling scheme for RSUs to provide a balance between 
serving downloads and upload requests from fast-moving vehicles on highways. An interesting paper by Eckhoff et al. propose to utilize parked vehicles as relay nodes to address the disconnected network problem. Extensive simulations and real life experiments show that parked cars can increase cooperative awareness by over $40 \%$ [18].

While the aforementioned studies shed light on the RSU placement problem and the communications between vehicles and the RSUs, it should be noted that, to the best of our knowledge, there are no existing studies on the use of vehicles as RSUs.

\section{CONCLusion}

In this paper, we propose a biologically-inspired new approach to implementing Roadside Units. Instead of using a costly roadside infrastructure (such as RSUs) or high-packet-latency Cellular networks and WiFi, we leverage the use of DSRC-equipped vehicles to serve as temporary roadside units. Based on the designed local rules, a DSRC-equipped vehicle independently determines whether it should serve as an RSU; and if so, it stops for a small duration and rebroadcasts the message. Results show substantial improvement in terms of message reachability which is crucial for safety message dissemination application in VANETs. It is worth mentioning that even though the benefits reported in this paper are based on a specific safety application (Post Crash Notification), our preliminary results show that the same concept could be used for other safety and efficiency applications of VANETs also.

While the solution proposed to the RSU deployment problem is interesting in itself, perhaps even a more interesting global conclusion is how the biologically inspired approach to solving fundamental transportation problems can be generalized and used as a powerful approach and tool for solving several important transportation problems. Our ongoing work is currently looking into other instances of the same approach for solving other outstanding transportation problems.

\section{ACKNOWLEDGMENT}

This research was supported in part by the T-SET University Transportation Center sponsored by the US Department of Transportation under Grant No. DTRT12-G-UTC11.

\section{REFERENCES}

[1] Department of Transportation, "ITS Cost Database (RS-TC and RS-I), http://www.benefitcost.its.dot.gov/ [Accessed on February 20, 2011]."

[2] W. Viriyasitavat and O. K. Tonguz, "Cars as roadside units: A cooperative solution," in Proc. of the IEEE Vehicular Technology Conference (VTC), pp. 1-5, September 2012.

[3] O. K. Tonguz and W. Viriyasitavat, "Transitioning To a Roadside Unit State, U.S. Patent Application no. PCT/US2013/022251,” July $25,2013$.

[4] M. Freitas, "Talk: Vehicle Infrastructure Integration," U.S. Department of Transportation, October 2005, http://www.vehicle-infrastructure.org/documents/VII\%20Traveler\%20Info\%20Overview.pdf.

[5] National Highway Traffic Safety Administration (NHTSA), U.S. Department of Transportation, "Frequency of Target Crashes for IntelliDrive Safety Systems," October 2010.

[6] U.S. Department of Transportation, "Intelligent Transportation Systems for Traveler Information: Deployment Benefits and Lessons Learned,” January 2007, http://ntl.bts.gov/lib/jpodocs/brochure/14319_files/14319.pdf.

[7] Transportation Research Board of The National Academies, "The RoadWay INFOstructure: What? Why? How?," Transportation Research Circular, November 2003, http://onlinepubs.trb.org/onlinepubs/circulars/ec057.pdf.

[8] O. K. Tonguz, "Biologically inspired solutions to fundamental transportation problems," IEEE Communications Magazine, vol. 49, pp. 106-115, November 2011 (the hypothesis that fundamental transportation problems can be solved via biologically inspired approaches was first formulated by O. K. Tonguz in the Keynote he delivered at the 2010 IEEE Vehicular Networking Conference in December 2010 in Jersey City, NJ). 
[9] W. Viriyasitavat, F. Bai, and O. K. Tonguz, "UV-CAST: An Urban Vehicular Broadcast Protocol," IEEE Communications Magazine, Special Issue on Automotive Network Series, vol. 49, pp. 116-124, November 2011.

[10] O. K. Tonguz, W. Viriyasitavat, and F. Bai, "Modeling urban traffic: a cellular automata approach," IEEE Communications Magazine, vol. 47, pp. 142-150, May 2009.

[11] W. Viriyasitavat, O. K. Tonguz, and F. Bai, "Dynamics of Network Connectivity in Urban Vehicular Networks," IEEE Journal on Selected Areas of Communications, Special Issue on Vehicular Communications and Networkings, vol. 29, pp. 515-533, March 2011.

[12] ZipCar, "http://www.zipcar.com/ [accessed on July 31, 2011]."

[13] N. Banerjee, M. D. Corner, D. Towsley, and B. N. Levine, "Relays, base stations, and meshes: enhancing mobile networks with infrastructure," in Proc. of the ACM international conference on Mobile computing and networking (MobiCom), pp. 81-91, 2008.

[14] O. Trullols, M. Fiore, C. Casetti, C. F. Chiasserini, and J. M. B. Ordinas, "Planning roadside infrastructure for information dissemination in intelligent transportation systems," Computer Communications, vol. 33, no. 4, pp. 432-442, 2010.

[15] J. Lee and C. Kim, "A roadside unit placement scheme for vehicular telematics networks," in Advances in Computer Science and Information Technology, vol. 6059 of Lecture Notes in Computer Science, pp. 196-202, Springer Berlin / Heidelberg, 2010.

[16] S.-Y. Wang, Y.-W. Cheng, C.-C. Lin, W.-J. Hong, and T.-W. He, "A vehicle collision warning system employing vehicle-to-infrastructure communications," in Proc. of the IEEE Wireless Communications and Networking Conference (WCNC), pp. 3075-3080, 2008.

[17] Y. Zhang, J. Zhao, and G. Cao, "On scheduling vehicle-roadside data access," in Proc. of the ACM international workshop on Vehicular ad hoc networks (VANET), pp. 9-18, 2007.

[18] C. Sommer, D. Eckhoff, and F. Dressler, "IVC in Cities: Signal Attenuation by Buildings and How Parked Cars Can Improve the Situation," IEEE Transactions on Mobile Computing, 2013. to appear.

\section{BIOGRAPHIES}

Ozan K. Tonguz is a tenured full professor in the Electrical and Computer Engineering Department of Carnegie Mellon University (CMU). He currently leads substantial research efforts at CMU in the broad areas of telecommunications and networking. He has published about 300 papers in IEEE journals and conference proceedings in the areas of wireless networking, optical communications, and computer networks. He is the author (with G. Ferrari) of the book Ad Hoc Wireless Networks: A Communication-Theoretic Perspective (Wiley, 2006). In December 2010, he founded the CMU startup known as Virtual Traffic Lights, LLC, which specializes in providing solutions to acute transportation problems using vehicle-to-vehicle and vehicle-to-infrastructure communications paradigms. His current research interests include vehicular networks, wireless networks, sensor networks, self-organizing networks, smart grid, bioinformatics, and security. He currently serves or has served as a consultant or expert for several companies, major law firms, and government agencies in the United States, Europe, and Asia.

Wantanee Viriyasitavat is a lecturer in the Faculty of Information and Communication Technology at Mahidol University, Bangkok, Thailand. During 2012-2013, she was a Research Scientist in the Department of Electrical and Computer Engineering at Carnegie Mellon University (CMU), Pittsburgh, PA. She received her B.S./M.S., and Ph.D. degrees in electrical and computer engineering from CMU in 2006 and 2012, respectively. Between 20072012, she was a Research Assistant at Carnegie Mellon University, where she was a member of General Motors Collaborative Research Laboratory (CRL) and was working on the design of a routing framework for safety and non-safety applications of vehicular ad hoc wireless networks (VANETs). Her current research interests include traffic mobility modeling, network connectivity analysis, and protocol design for wireless ad hoc networks. 